\title{
Successful Treatment of Brainstem Blastomycosis with Fluconazole
}

\author{
Katherine E. Brick, MD and William A. Agger, MD
}

\begin{abstract}
The lipid formulation of amphotericin B is the initial drug of choice for central nervous system blastomycosis, but it is costly and associated with significant toxicity. This case report details a patient with primary pulmonary blastomycosis with dissemination to the skin, one joint, and the brainstem that was successfully treated solely with high-dose fluconazole.
\end{abstract}

Keywords: Blastomycosis; Central nervous system; Fluconazole

Corresponding Author:

William A. Agger, MD

Infectious Disease Section

Gundersen Lutheran Health System

1900 South Avenue

La Crosse, WI 5460

Tel: (608) $782-7300$

Fax: (608) 775-5542

Email: waagger@gundluth.org

Received: May 17, 2011

Revised: June 2I, 201I

Accepted: July 6, 2011

doi: $10.3121 / \mathrm{cmr} .2011 .1023$
$B$ lastomyces dermatitidis is a dimorphic fungal pathogen endemic to eastern North America that infects humans and animals. Following inhalation, dissemination can occur to skin, bone, the genitourinary tract, and the central nervous system (CNS), in decreasing order of frequency, ${ }^{1}$ and is reported in both immunosuppressed and immunocompetent hosts. With a reported mortality rate of $18 \%$ despite appropriate therapy, CNS involvement is a rare but serious complication. ${ }^{2}$ Over the past 10 years, the lipid formulation of amphotericin $\mathrm{B}$ has emerged as the initial drug of choice for CNS blastomycosis, ${ }^{3}$ with monotherapy azole use predominant among isolated pulmonary cases and mild to moderate, non-CNS dissemination. We report a case of blastomycosis disseminated to the skin, one joint, and the brainstem that was treated unconventionally, yet successfully, with high-dose fluconazole alone.

\section{Case Report}

A 29-year-old Caucasian man with a history of logging in rural Wisconsin was referred for an Infectious Disease consultation after a 2-week-long illness. Physical examination demonstrated normal vital signs, lungs clear to auscultation, and no hepatosplenomegaly. Abnormalities on examination included pain and swelling in his right knee, an inability to fully retract the left eyelid, and edema and strabismus of the left eye with medial deviation. He also had an erythematous exophytic verrucous plaque above the right brow, swelling and erythema around the left orbit, and scattered erythematous papules above the left oral commissure (figure 1), as well as erythematous plaques at the anterior costal margin and right tibial tuberosity. A potassium hydroxide preparation of the left upper lip lesion demonstrated broad-based budding yeast consistent with Blastomyces dermatitidis. Histologic analysis of a $6 \mathrm{~mm}$ punch biopsy specimen showed dermal granulomatous and suppurative inflammation with microabscess formation containing numerous broad-based budding yeasts typical of $B$. dermatitidis and pseudoepitheliomatous hyperplasia. Notable laboratory results included a white 


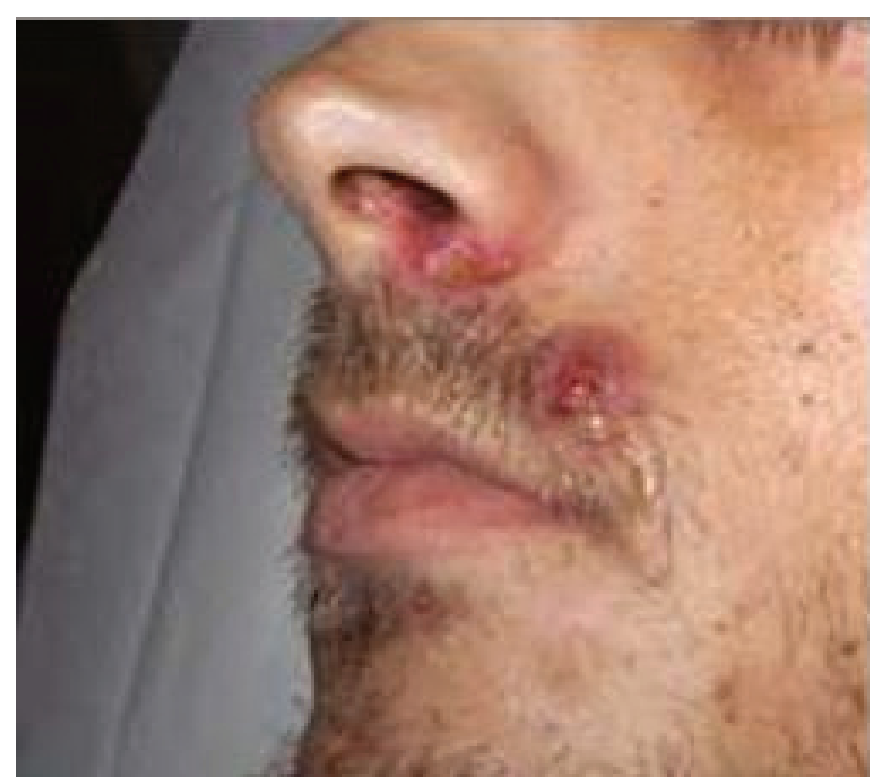

Figure 1. Photograph demonstrating erythematous, pustular blastomycotic papule.

blood cell count of $11,000 / \mu \mathrm{L}$, urinalysis with 50 white blood cells per high-power field and $>100$ bacteria with a negative culture, normal creatinine level, and liver function test results within reference range. Results of laboratory tests for rheumatoid factor, antinuclear antibody, and human immunodeficiency virus (HIV) later returned negative, and bacterial and fungal blood cultures were negative for growth. Results of serological tests for antibody to the A antigen of $B$. dermatitidis were positive, including immunodiffusion and a complement fixation of 1:32. Results of serological tests for coccidioidal immunoglobulin $\mathrm{G}$ and immunoglobulin $\mathrm{M}$, cryptococcal antigen, histoplasma immunodiffusion, yeast, and mycelial forms were all negative.

The patient was admitted to the hospital that evening with persistent diplopia and the aforementioned skin lesions. Chest radiography revealed a small, left upper lobe infiltrate, although his lungs were clear to auscultation. He was started on high-dose (800 mg daily) intravenous fluconazole and underwent a magnetic resonance imaging (MRI) scan of the brain. The imaging showed a $6 \mathrm{~mm}$ superior brainstem lesion without evidence of mass effect (figure 2). Following extensive discussion with the patient, he elected - against medical advice - to receive fluconazole rather than the recommended amphotericin $\mathrm{B}$.

While hospitalized, he experienced gradual improvement of his ophthalmoplegia and cutaneous lesions on the high-dose fluconazole treatment. Repeat intracranial imaging was not obtained, but because the patient showed complete neurologic improvement after 7 days, he was discharged with a peripherally inserted central catheter in place and received a total 21-day course of intravenous fluconazole. He was followed in the Infectious Disease clinic with continued resolution of his skin lesions, was switched to oral fluconazole
$600 \mathrm{mg}$ daily, and was seen monthly for the next 6 months. After 6 months of therapy his chest radiograph was repeated and revealed only residual, stable fibrotic changes at the left upper lobe. The brain MRI was not repeated given complete resolution of neurologic symptoms and due to patient refusal. He completed 6 months of fluconazole therapy and has not had recurrence of symptoms over the last 6 years.

\section{Discussion}

Medications used to treat blastomycosis with CNS involvement must cross the blood-brain barrier; therefore, recent guidelines published by the Infectious Diseases Society of America have recommended treatment with the lipid formulation of amphotericin B for 4 to 6 weeks or until CNS symptoms have improved, followed by fluconazole, itraconazole, or voriconazole for an additional 10 to 11 months. ${ }^{4}$ The lipid formulation has replaced the more nephrotoxic deoxycholate formulation, but it still has significant electrolyte disturbances and renal toxicity, which often limits its duration of use. ${ }^{5,6}$ High-dose fluconazole (greater than $400 \mathrm{mg}$ and preferably $800 \mathrm{mg}$ ) has been used as a treatment option in patients with less severe, non-CNS blastomycosis. ${ }^{7}$ While fluconazole has less activity than itraconazole against blastomycosis in vitro, it does have superior CNS penetration, ${ }^{8}$ accounting for its use following control of CNS disease with amphotericin B.

As suggested by the practice guidelines on treatment of blastomycosis, ${ }^{4}$ initial monotherapy with high-dose fluconazole may have a role in the treatment of CNS blastomycosis, but clinical experience has been sparse because the lipid formulation of amphotericin B has been almost exclusively used to control acute disease prior to long-term azole therapy. Two earlier case reports used lower dose

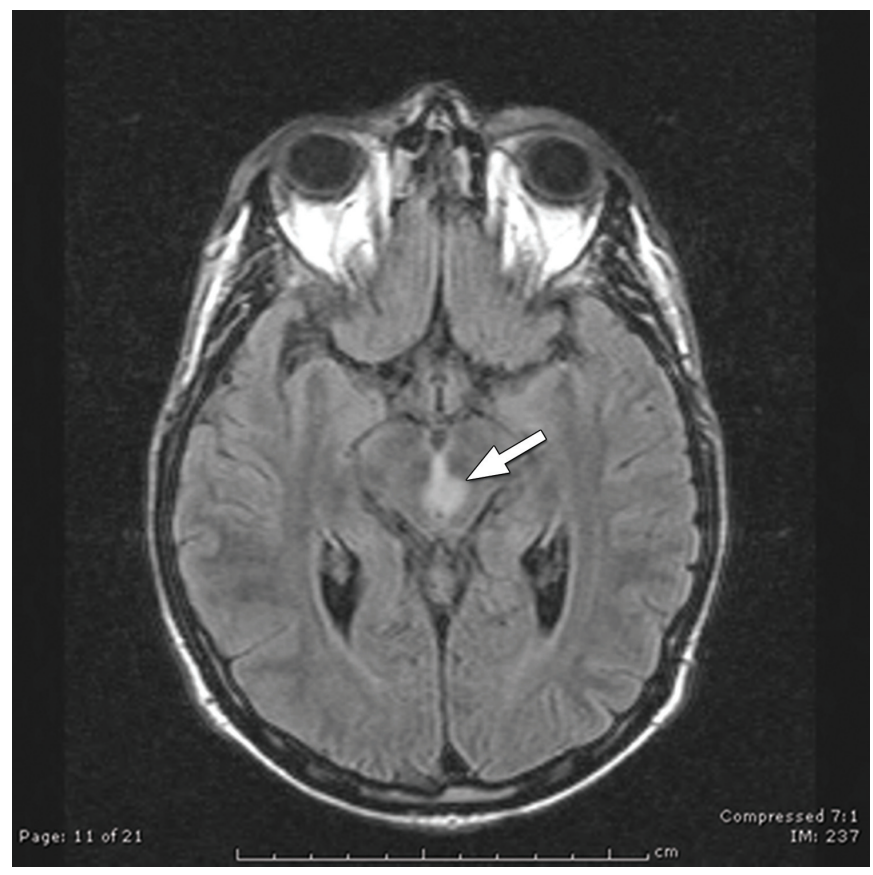

Figure 2. Magnetic resonance imaging scan without contrast showing central brainstem lesion (arrow). 
fluconazole successfully without prior amphotericin $\mathrm{B} ; ;^{9,10}$ however, the first case was based on suggestive CNS symptoms in the absence of imaging confirmation, with a dose of $200 \mathrm{mg}$ twice per day for 9 months, resulting in complete resolution of symptoms. ${ }^{9}$ The second case described an effective combination of fluconazole with flucytosine. ${ }^{10} \mathrm{~A}$ single case report also details efficacy of high-dose voriconazole for CNS blastomycosis infection in a patient where significant nephrotoxicity and drug intolerance precluded use of both formulations of amphotericin as well as itraconazole. ${ }^{11}$

Blastomycosis can recur despite treatment with amphotericin $\mathrm{B}$, but recurrence is usually related to an inadequate duration of subsequent azole use. For example, CNS blastomycosis developed in an immunocompetent adolescent patient 18 months after treatment for isolated pulmonary blastomycosis. At the time of initial diagnosis he did not have neurologic symptoms or other evidence of dissemination; thus, intracranial imaging was not performed, and he was treated with 2 weeks of amphotericin B deoxycholate followed by just 6 weeks of oral fluconazole. ${ }^{12}$ Such cases highlight the importance of adherence to the entire recommended duration of therapy (6 to 12 months in most cases), regardless of whether amphotericin or an azole is used initially. Given our patient's history of wood-cutting and the small infiltrate seen on chest radiograph, his CNS dissemination likely occurred following a subacute pneumonia that initially went unrecognized.

Our patient showed considerable and rapid improvement of his cutaneous disease, which correlated well with the improvement of his CNS symptoms; thus, it was warranted to continue fluconazole alone. In retrospect, he should have been treated with a full year of oral fluconazole, especially after the lack of amphotericin B initially. However, after 6 months of reliable monthly follow-up visits, he did not return to the Infectious Disease clinic as requested. The patient's urgent care and primary care visits over the past 6 years, as documented in his medical record, were reviewed and contain no evidence of recurrent signs or symptoms of infection.

It is widely agreed that high-dose fluconazole alone is not the standard of care for CNS blastomycosis, but amphotericin B does carry significant side effects that are often dose-limiting. The lipid formulation is also quite expensive. In the appropriate setting, and in a stable patient who is hospitalized and closely monitored for signs of neurologic improvement, high-dose fluconazole may be effective. Although we do not recommend routine use of high-dose fluconazole alone for CNS blastomycosis, we report its successful use in a patient for whom amphotericin B was not a viable option.

\section{References}

1. Bradsher RW, Chapman SW, Pappas PG. Blastomycosis. Infect Dis Clin North Am 2003;17:21-40,vii.
2. Bariola JR, Perry P, Pappas PG, Proia L, Shealey W, Wright PW, Sizemore JM, Robinson M, Bradsher RW Jr. Blastomycosis of the central nervous system: a multicenter review of diagnosis and treatment in the modern era. Clin Infect Dis 2010;50:797-804.

3. Cook PP. Amphotericin B lipid complex for the treatment of recurrent blastomycosis of the brain in a patient previously treated with itraconazole. South Med J 2001;94:548-549.

4. Chapman SW, Dismukes WE, Proia LA, Bradsher RW, Pappas PG, Threlkeld MG, Kauffman CA; Infectious Diseases Society of America. Clinical practice guidelines for the management of blastomycosis: 2008 update by the Infectious Diseases Society of America. Clin Infect Dis 2008;46: 1801-1812.

5. Gallis HA, Drew RH, Pickard WW. Amphotericin B: 30 years of clinical experience. Rev Infect Dis 1990;12:308-329.

6. Wong-Beringer A, Jacobs RA, Guglielmo BJ. Lipid formulations of amphotericin B: clinical efficacy and toxicities. Clin Infect Dis 1998;27:603-618.

7. Pappas PG, Bradsher RW, Kauffman CA, Cloud GA, Thomas CJ, Campbell GD Jr, Chapman SW, Newman C, Dismukes WE. Treatment of blastomycosis with higher doses of fluconazole. The National Institute of Allergy and Infectious Diseases Mycoses Study Group. Clin Infect Dis 1997;25:200-205.

8. Grant SM, Clissold SP. Fluconazole. A review of its pharmacodynamic and pharmacokinetic properties, and therapeutic potential in superficial and systemic mycoses. Drugs 1990;39:877-916.

9. Pearson GJ, Chin TW, Fong IW. Case report: treatment of blastomycosis with fluconazole. Am J Med Sci 1992;303:313-315.

10. Taillan B, Ferrari E, Cosnefroy JY, Gari-Toussaint M, Michiels JF, Paquis P, Lefichoux Y, Dujardin P. Favourable outcome of blastomycosis of the brain stem with fluconazole and flucytosine treatment. Ann Med 1992;24:71-72.

11. Bakleh M, Aksamit AJ, Tleyjeh IM, Marshall WF. Successful treatment of cerebral blastomycosis with voriconazole. Clin Infect Dis 2005;40:e69-e71.

12. Panicker J, Walsh T, Kamani N. Recurrent central nervous system blastomycosis in an immunocompetent child treated successfully with sequential liposomal amphotericin B and voriconazole. Pediatr Infect Dis J 2006;25:377-379.

\section{Author Affiliations}

Katherine E. Brick, MD*; William A. Agger, $M D^{\dagger}$

*Department of Medical Education, Gundersen Lutheran

Medical Foundation, La Crosse, Wisconsin, USA

†Section of Infectious Disease, Gundersen Lutheran Health

System, La Crosse, Wisconsin, USA 